\title{
Utilisation d'un nouveau bio-floculant extrait de cactus marocain dans le traitement des rejets chargés de chrome (VI) par le procédé de coagulation floculation
}

\author{
Aziza ABID, Abdeljalil ZOUHRI* et Abdelali IDER \\ Laboratoire des procédés de valorisation des ressources naturelles, des matériaux et \\ environnement, Département de chimie appliquée et environnement, Faculté des \\ sciences et techniques de Settat, BP 577 Maroc
}

*Correspondance,courriel : abdzouhri@yahoo.fr

\section{Résumé}

Dans ce travail, nous avons utilisé un nouveau floculant organique biodégradable extrait de jus de cactus (figuiers de barbarie, voir photo ci-dessous), dans un procédé physicochimique (coagulation floculation) afin de traiter des rejets liquides chargés en chrome (VI). Ce bio-floculant nous a permis de réduire la turbidité d'un effluent industriel, en passant de 100 NTU à des valeurs au dessous de 2 NTU. L'étude a été réalisée sur des échantillons pseudo-industriels préparés au laboratoire et des échantillons industriels issus d'une unité de traitement de surface (chromage).

L'étude comparative entres le floculant organique et le floculant industriel PPRQESTOL ${ }^{R}$ 2515.TR (floculant à base d'acrylamide et acrylate de sodium), a montré que notre biofloculant aboutit à des résultats satisfaisants au niveau du pouvoir de floculation. Une neutralisation ef coagulation avec la chaux, suivi par une floculation puis une décantation, pour la solution du chrome (VI) réduit en chrome (III) a montré un effet très significatif sur l'abattement de la turbidité et un pourcentage de rétention de chrome (VI) de plus de $99,5 \%$. Les résultats obtenus sont très encourageant et incitent notre équipe de recherche à développer cet axe de recherche afin de mettre en place des procédés alternatifs propres pour le traitement des eaux d'une manière générale et qui en même temps permettent la valorisation des ressources naturelles nationales.

Mot-clés : Effluent Industriel, chrome (VI), Turbidité, cactus, bio- floculant, Traitement, coagulation floculation. 


\section{Abstract \\ Use of a new bio-flocculent (extracted from prickly pear/Moroccan cactus) in the treatment of rejections charged with Chromium (VI) by the coagulation flocculation process.}

In this work, we used a new biodegradable organic flocculating agent we extracted from the juice of prickly pear, in a physicochemical process (coagulation- flocculation) in order to treat liquid rejections charged with chromium (VI). This bioflocculent helps reduce the turbidity of industrial effluents, making it pass from 100 NTU to values below 2 NTU. The tests were carried out on pseudo-industrial samples prepared at the laboratory and samples from a surface treatment unit.

The comparative study of the organic flocculating agent with the industrial flocculating agent PPRQESTOL ${ }^{R}$ 2515.TR (containing acrylamide and acrylate of sodium), showed the bioflocculent's very good competitiveness and its strong capacity of flocculation. Neutralization and coagulation with lime, followed by flocculation then decantation, for the solution of chromium (VI) reduced to chromium (III), showed a very significant effect on the abatement of turbidity and a percentage of chromium (VI) retention of more than $99,5 \%$. This work brings to satisfactory results which incite our team to further research in order to boost our national natural resources by using alternate proper processes for water treatment.

Keywords : Industrial effluent, chromium (VI), turbidity, cactus, bio-flocculating agent, treatment, coagulation-flocculation.

\section{Introduction}

L'environnement et les équilibres naturels sont à la base du développement social et humain. Dans ce cadre et pour une vraie stratégie de développement durable de l'environnement dans son double aspect; gestion des ressources naturelles et des différents rejets, on doit rassembler nos efforts pour la valorisation des ressources naturelles $[1,2,10]$. Afin de répondre à ces objectifs, nous avons étudié la possibilité de valoriser un produit actif d'une plante naturelle (Photo $I$ ci-dessous) en tant que floculant naturel, utile dans le traitement physico-chimique "coagulation-floculation" des effluents liquides.

Dans cette optique de traitement de ces rejets, de nombreux travaux $[3,4,7,9,13$.] ont été effectués afin d'éliminer les ions métalliques par voix physicochimiques. Les résultats 
obtenus étaient assez satisfaisants, mais les procédés suivis sont plus au moins compliqués et nécessitent l'utilisation de nombreux produits chimiques en plusieurs étapes. Nous avons proposé ce travail pour l'amélioration de la technique coagulation floculation; d'une part en simplifiant le nombre des étapes suivies et d'autre part en introduisant un nouveau floculant.

Nous envisageons également de valoriser les substances naturelles de notre région (centre et sud du Maroc), minimiser l'effet toxique des produits chimiques (chlorure de fer et sulfate d'alumine), respecter les normes de plus en plus rigoureuses $[4,5]$ et réduire le coût de traitement des effluents industriels. Dans ce contexte, nous avons mis en place des procédés d'élimination des ions métalliques par association des deux étapes de neutralisation et de coagulation par la chaux en utilisant comme agent floculant le nouveau produit extrait des cactus marocains " figuier de barbarie". L'objectif de notre travail, été d'une part la mise en évidence de l'efficacité d'un nouveau floculant naturel biodégradable grâce à une étude comparative avec un floculant usuel à base d'acrylamide [2] très utilisé dans le traitement des rejets industriels et d'autre part le traitement physico-chimique "coagulation-floculation" des eaux résiduaires pseudo industrielle et industrielle chargées de chrome (VI).

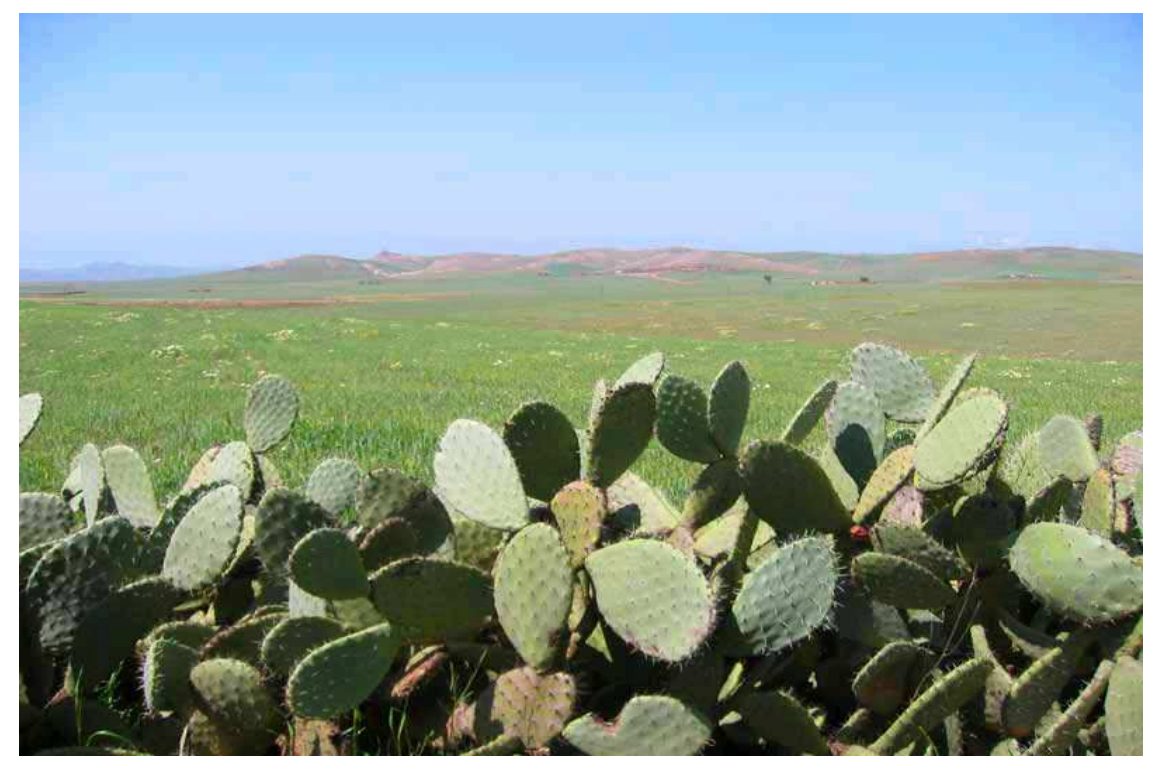

Photo 1 : d'un champ de cactus (figuiers de barbarie), situé entre Casa et Marrakech (http://loindetout.free.fr/images/photos/maroc2003/maroc4.jpg) 


\section{Matériels et méthodes}

Le floculant organique (bio-organique) est extrait du cactus qui pousse dans le centre du Maroc. Le produit à l'état naturel est un liquide visqueux de coloration verte, de $\mathrm{pH}=$ 6.5 , miscible à l'eau, sa masse volumique est de $1,008 \mathrm{~kg} / \mathrm{l}$. Son analyse réalisée par spectroscopie UV a donné le spectre ci-dessous (Figure I).

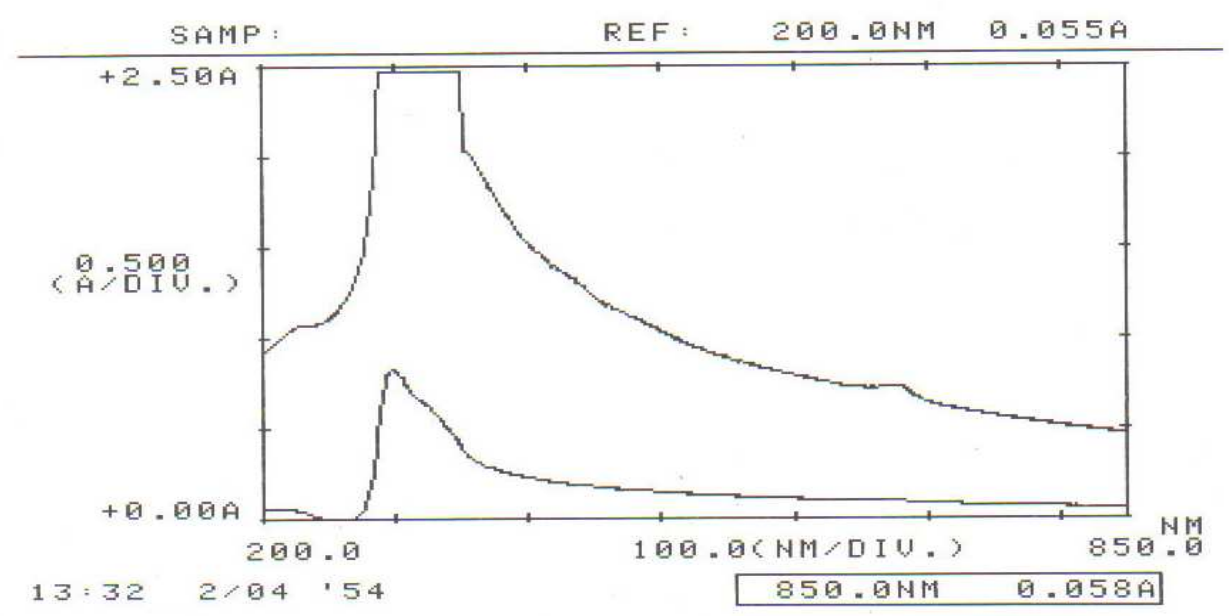

Figure 1 : Spectres d'absorbance UV de jus de cactus vers $300 \mathrm{~nm}$. Jus à $100 \%$ (pic en haut), jus à $10 \%$ dans l'eau (pic en bas).

Dans ce travail, l'extraction du floculant organique a été réalisé selon les étapes suivantes:

- Broyage du cactus après nettoyage,

- Extraction du jus de cactus par filtration au tamisage,

- Dilution de jus dans de l'eau à $10 \%$ et homogénéisation par agitation pendant 15 à 20 minutes.

Le mélange obtenu est relativement stable, il peut conserver sa capacité de floculation pendant plusieurs jours en dehors de tout système de conservation.

\section{Le matériel utilisé est le suivant :}

- Turbidimètre de marque HANNA LP2000-11

- pH-mètre Accumet Basic de marque AB15 
- Système Jar-test (Model ISCO RPM/OPM).

- Spectrophotomètre UV-Visible, type UV-160 mode SHIMADZU

Les essais de traitement ont été effectués à température ambiante.

\section{Résultats et discussion}

\section{3-1. Evaluation du pouvoir floculant de jus de cactus}

Afin de comparer le pouvoir floculant de notre réactif avec d'autres qui sont couramment utilisés, nous avons dans un premier temps, mené notre étude sur une solution pseudoindustrielle à $100 \mathrm{ppm}$ en $\mathrm{Cr}(\mathrm{VI})$. Au cours de cette première étape, la formation des flocs, de leurs tailles ainsi que l'évolution de la concentration en chrome ont été suivies (Tableaux 1 et 2). L'abattement de la turbidité a été également suivi (Figure 2). Les essais de coagulation floculation ont été menés par le système Jar-test selon les étapes suivantes:

- Ajustement de pH à 2, suivi d'une réduction du $\mathrm{Cr}$ (VI) au $\mathrm{Cr}$ (III)

- Ajustement de pH à 9 selon les 6 essais décrits dans les Tableaux 1 et 2,

- Ajout de coagulant et/ou floculant sous une agitation de 100 tr/min pendant 1 à $2 \mathrm{mn}$, puis une agitation de $40 \mathrm{tr} / \mathrm{mn}$ pendant $15 \mathrm{mn}$ préalablement déterminées.

- Précipitation puis analyse du résidu de chrome en solution sous forme de $\mathrm{Cr}(\mathrm{VI})$.

Tableau 1 : Effet des différents ajouts sur la floculation et l'élimination du chrome en présence de la chaux : Cas d'une solution pseudo-industrielle à 100 ppm en Cr(VI)

\begin{tabular}{|c|c|c|c|c|}
\hline $\mathbf{N}^{\circ}$ d'essai & 1 & 2 & 3 & 4 \\
\hline $\begin{array}{l}\text { Réactifs } \\
\text { utilisés }\end{array}$ & $\begin{array}{c}\text { Chaux }+2 \mathrm{ml} \text { de } \\
\text { jus de cactus } \\
\text { dilué }\end{array}$ & $\begin{array}{l}\text { Chaux }+\mathrm{FeCl}_{3} \\
+2 \mathrm{ml} \mathrm{de} \mathrm{jus} \mathrm{de} \\
\text { cactus dilué }\end{array}$ & $\begin{array}{l}\text { Chaux }+0.1 \mathrm{ml} \\
\text { de floculant } \\
\text { industriel } \\
\text { à } 1 \%\end{array}$ & $\begin{array}{c}\text { Chaux }+\mathrm{FeCl}_{3} \\
+0.1 \mathrm{ml} \mathrm{de} \\
\text { floculant - industriel } \\
\text { à } 1 \%\end{array}$ \\
\hline Observation & bonne floculation & bonne floculation & bonne floculation & bonne floculation \\
\hline$[\mathrm{Cr}(\mathrm{VI})] \mathrm{ppm}$ & 0,3 & 0,27 & 0,25 & 0,32 \\
\hline
\end{tabular}


Tableau 2 : Effet des différents ajouts sur la floculation et l'élimination du chrome en présence de la soude : Cas d'une solution pseudo-industrielle à 100 ppm en $\mathrm{Cr}(\mathrm{VI})$

\begin{tabular}{|c|c|c|}
\hline $\mathbf{N}^{\circ}$ d'essai & $\mathbf{5}$ & $\mathbf{6}$ \\
\hline Réactifs utilisés & $\begin{array}{c}\mathrm{NaOH}+\mathrm{FeCl}_{3}+2 \mathrm{ml} \mathrm{de} \\
\text { jus de cactus dilué }\end{array}$ & $\begin{array}{c}\mathrm{NaOH}+\mathrm{FeCl}_{3}+0.1 \mathrm{ml} \mathrm{de} \\
\text { Floculant-industriel à } 1 \%\end{array}$ \\
\hline Observation & bonne floculation & floculation moyenne \\
\hline$[\mathrm{Cr}(\mathrm{VI})] \mathrm{ppm}$ & 0,4 & 0,3 \\
\hline
\end{tabular}

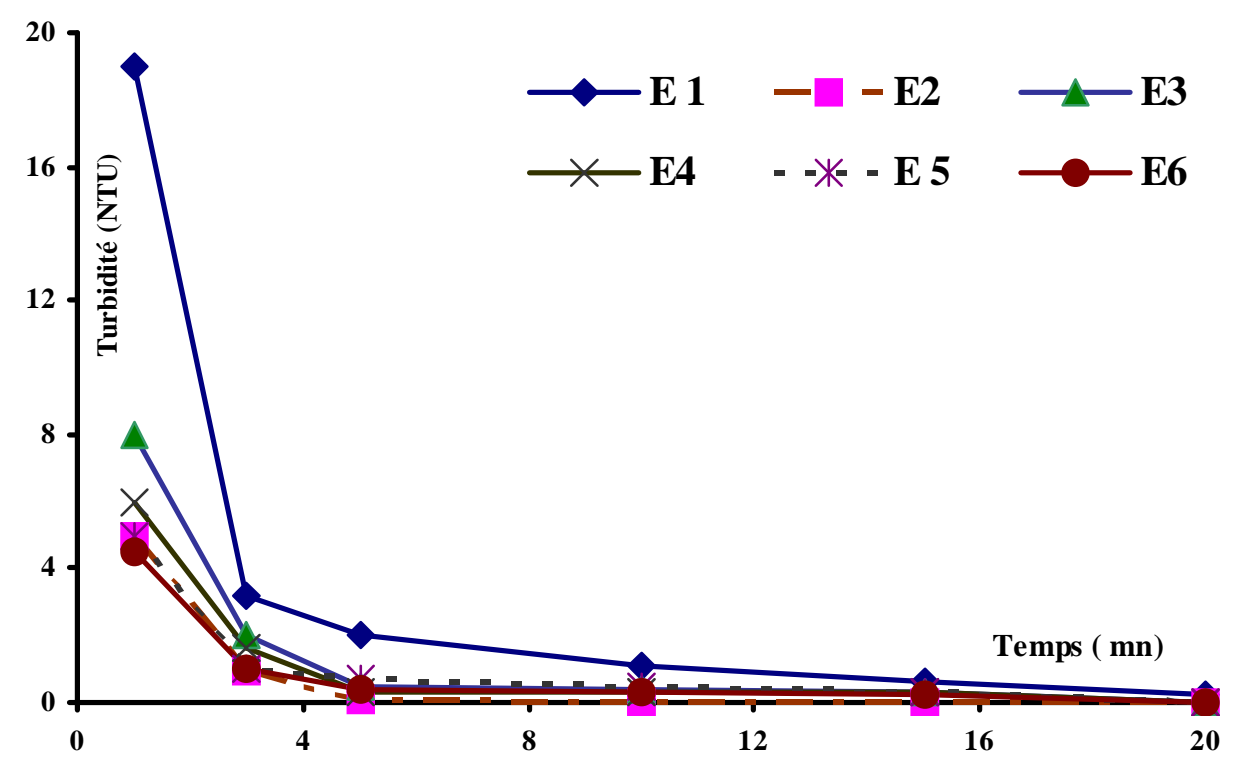

Figure 2 : Suivi de l'abattement de la turbidité pour les 6 essais en fonction du temps de la sédimentation : Cas d'une solution pseudo-industrielle à 100 ppm en (r(VI)

Pour les essais (1, 2, 3 et 4) ont été réalisés soit en présence de la chaux seule ou avec de la chaux et le chlorure de fer, les résultats obtenus, montrent une très bonne et immédiate floculation de même ordre de grandeur pour les deux floculants.

Quant aux essais (5 et 6 ) nous avons utilisé la soude en tant que neutralisant et le chlorure de fer comme coagulant, la floculation est moins bonne pour le produit industriel 
(essai 5) alors que pour le produit bio-organique, la floculation et fortement importante (essai 6).

L'ensemble des essais, montre donc que le jus de cactus présente une très bonne capacité de floculation en présence de la chaux seule ou mélangée avec le chlorure de fer. L'efficacité de ce liquide est comparable à celle des floculants industriels tel que le PPRQESTOL ${ }^{R}$ 2515.TR après neutralisation et coagulation avec la chaux.

3-2. Traitement des rejets industriels chromés issus d'une unité de traitement de surface (chromage)

Nous avons mené notre étude sur 2 échantillons issus d'un rejet industriel à différentes teneurs en $\mathrm{Cr}(\mathrm{VI})$ à $22 \mathrm{ppm}$ et $97 \mathrm{ppm}$.

Les concentrations en $\mathrm{Cr}$ (VI) avant et après traitement sont représentées dans le (Tableau 3). L'évolution de la turbidité (Figures 3 et 4) et les absorbances (Figures 5 et 6)ont été également suivies.

Tableau 3 : Echantillons industriels chargés en chrome (VI) traités par le floculant organique et le floculant industriel

\begin{tabular}{|c|c|c|c|c|}
\cline { 2 - 5 } \multicolumn{1}{c|}{} & \multicolumn{2}{c|}{ Echantillon I } & \multicolumn{2}{c|}{ Echantillon 2 } \\
\cline { 2 - 5 } \multicolumn{1}{c|}{} & floculant industriel & $\begin{array}{c}\text { floculant } \\
\text { organique }\end{array}$ & $\begin{array}{c}\text { floculant } \\
\text { industriel }\end{array}$ & $\begin{array}{c}\text { floculant } \\
\text { organique }\end{array}$ \\
\hline $\begin{array}{c}{[\mathrm{Cr}(\mathrm{VI})] \text { en ppm avant }} \\
\text { traitement }\end{array}$ & 22 & 22 & 97 & 97 \\
\hline $\begin{array}{c}{[\mathrm{Cr}(\mathrm{VI})] \text { en ppm après }} \\
\text { traitement }\end{array}$ & 0,35 & 0,3 & 0,5 & 0,33 \\
\hline
\end{tabular}




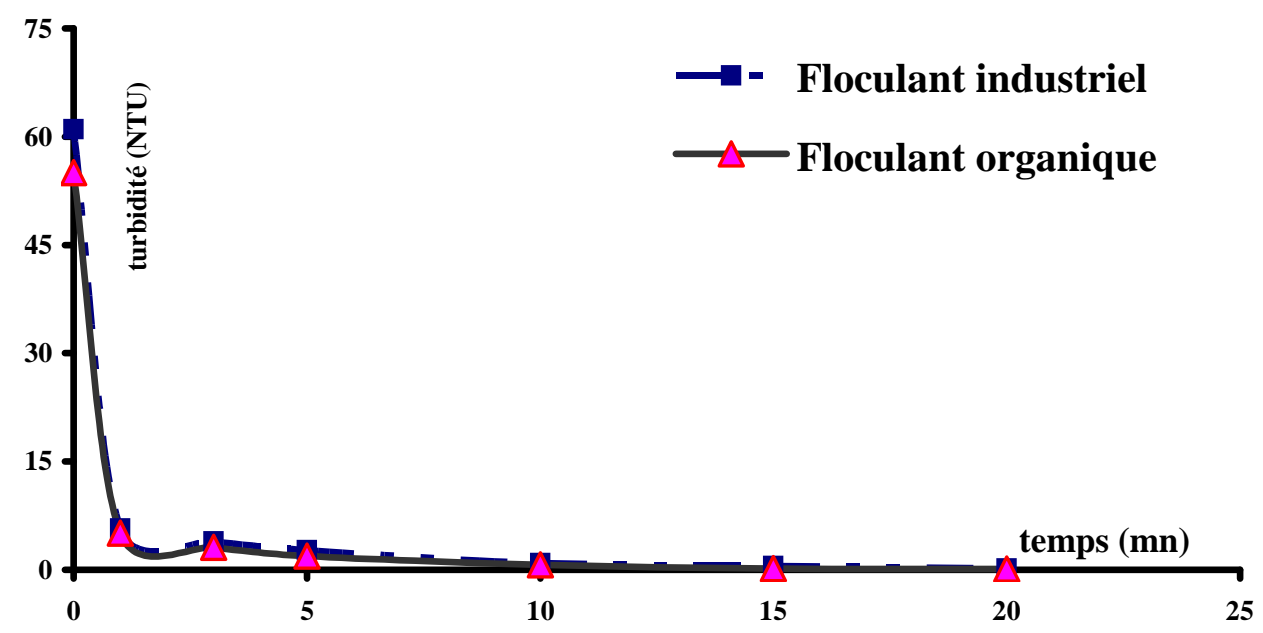

Figure 3 : Evolution de la turbidité en fonction du temps de sédimentation : Cas de l'échantillon I (97 ppm en (r(VI)) traité par les deux floculants

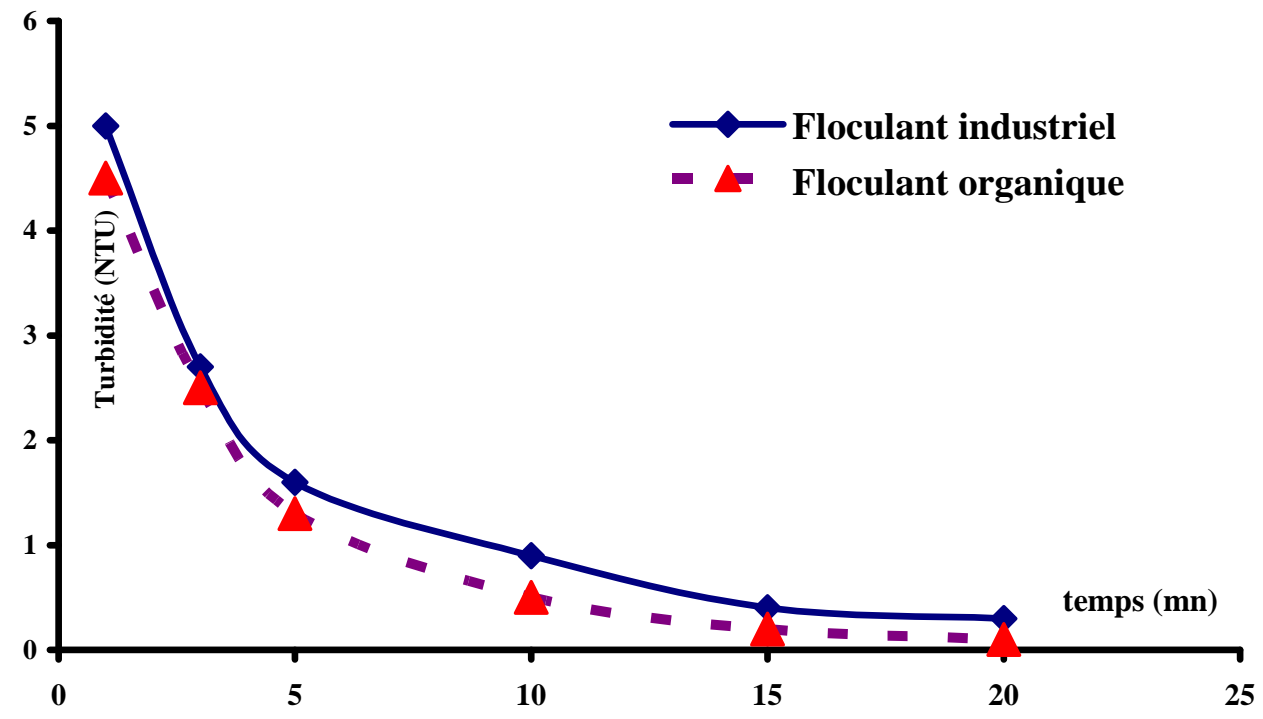

Figure 4 : Evolution de la turbidité en fonction du temps de sédimentation Cas de l'échantillon 2 (22 ppm en (r(VI)) traité par les deux Floculants 


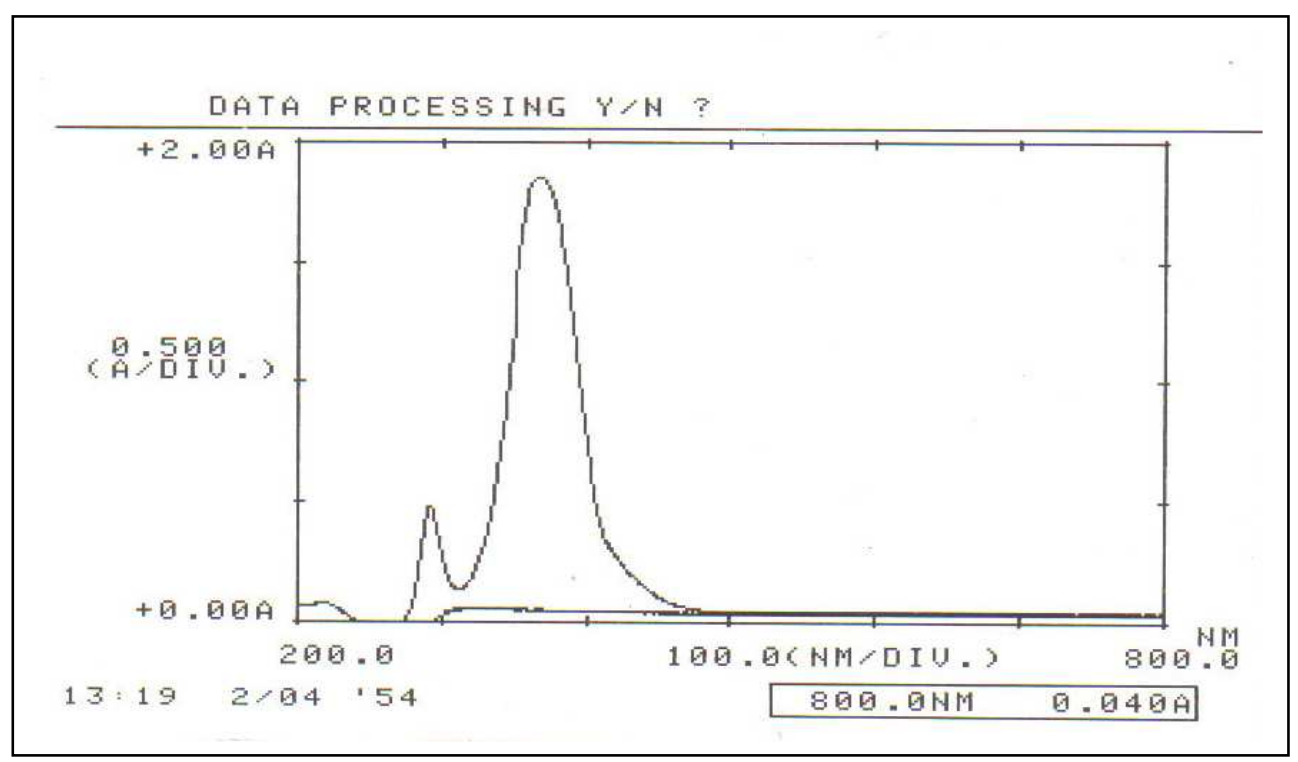

Figure 5 : Spectre UV de l'échantillon industriel(97 ppm en (r(VI)) avant et après traitement avec le floculant industriel

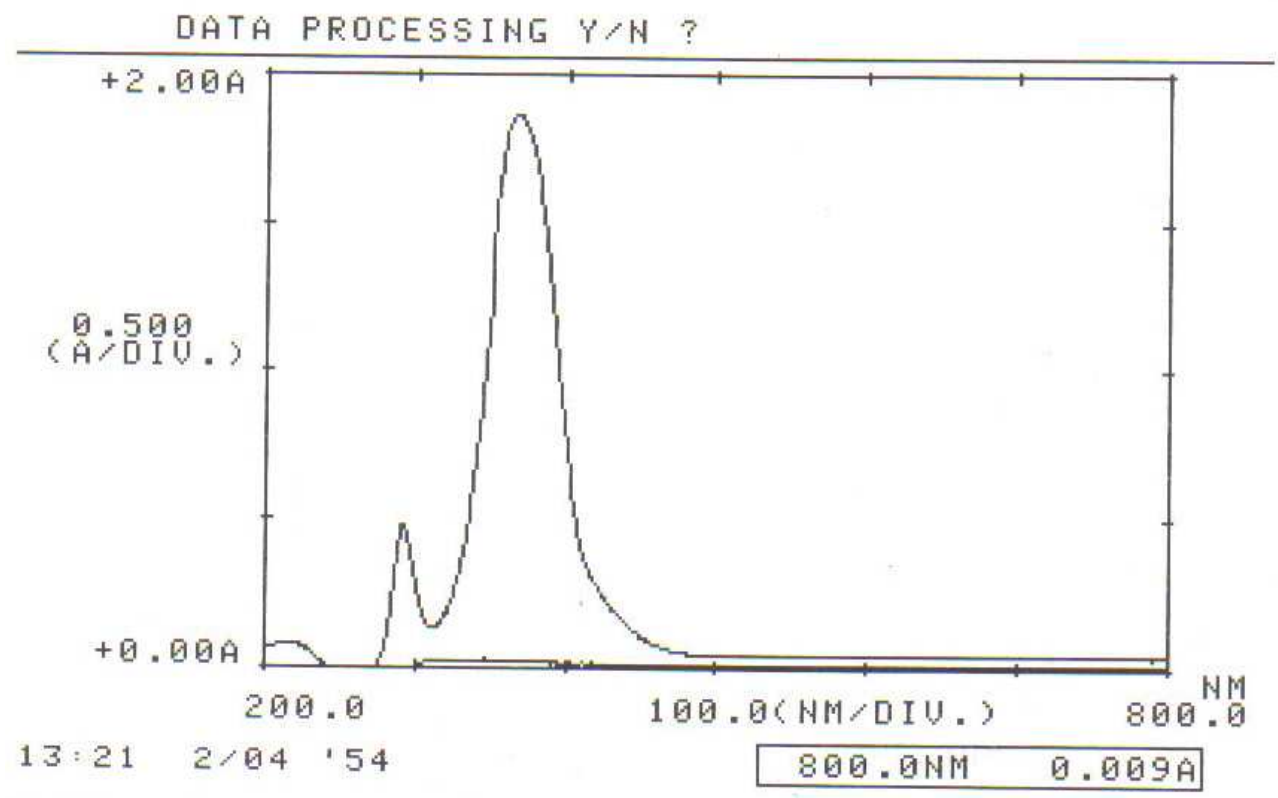

Figure 6 : Spectre UV de l'échantillon industriel(97 ppm en Cr(VI)) avant et après traitement avec le floculant bio-organique 
D'après les Figures 3 et 4 , on constate que l'efficacité du floculant organique vis-à-vis de l'abattement de la turbidité est légèrement signifiante par rapport à celle obtenue dans le cas du floculant industriel PPRQESTOL ${ }^{R} 2515$.TR. Nous constatons également que le pourcentage de rétention des deux floculants est de 98.50 à 99.50 .

D'après les Figures 5 et $\boldsymbol{6}$, on voit très bien que les complexes formés par les deux floculants et le chrome absorbent dans la même zone $(370-380 \mathrm{~nm})$ alors que dans le cas de jus cactus l'absorbance est de l'ordre $300 \mathrm{~nm}$. Ce qui traduit une interaction; jus de cactus-chrome (VI).

Les résultats relatifs à l'abattement de la turbidité présentés sur les deux dernières figures, sont en nette cohérence avec les spectres UV présentant une très faible absorbance dans le cas de l'échantillon traité.

On peut noter que le floculant bio-organique est relativement avantageux par rapport au floculant industriel.

\section{Conclusion}

Ce travail présente une étude originaire, car il permettra : D'une part, une contribution intéressante dans le domaine de la valorisation des ressources naturelles de notre pays. D'autres part, il permet la possibilité d'introduire un nouveau réactif biodégradable dans le processus de traitement physico-chimique par procédé de coagulation floculation. Nous espérons par ce travail remplacer certains coagulants inorganiques largement appliqués dans le domaine de traitement des eaux et ayant des inconvénients sur l'environnement et en particulier la santé humaine.

Les avantages d'un traitement avec le jus de cactus (figuier de barbarie) seront nombreux. De plus, ce procédé sera rentable et s'introduira dans le développement durable dans les régions ou les cactus sont cultivés à grande échelle. Parmi les autres avantages, on peut éviter des modifications relatives aux propriétés physico-chimiques de l'échantillon traité ainsi que la bove récupérée sera biodégradable et exempte de fer ou d'aluminium et de polymères chimiques. Le jus de cactus aura donc une forte possibilité d'être une alternative aux coagulants ef floculants chimiques. 


\section{Références}

[1] - JINGDONG ZHANG, FANG ZHANG, YUNHONG LUO, HONG YANG, "A preliminary study on cactus as coagulant in water treatment", Process Biochemistry, 41 (2006) 730-733.

[2] - A. DIAZ, N. RINCON, A. ESCORIHUELA, N. H. FERNANDEZ, E. CHACIN, CF. FOTER."A preliminary evaluation of turbidity removal by natural coagulante indigenous to venzuela", Process Biochemistry 35 (1999) 391-395.

[3] - F. EDELINE, L'épuration physico-chimique des eaux, CEBEDOC $4{ }^{\text {ème }}$ édition (1993)

[4] - J. BONTOUX, Introduction à l'étude des eaux douces, édition CEBEDOC (1993)

[5] - MINISTERE de l'aménagement du territoire de l'eau et e l'environnement, Les nouvelles lois de protection de l'environnement, Maroc, (2003)

[6] - J. RODIER, Analyse de l'eau, 8 éme édition (1996)

[7] - A. ZOUHRI, II Utilisation de l'éther couronneDC18-6 pour la récupération sélective de l'acide chromique à partir des effluents de traitement de surface (chromage) II, Doctorat de L'UIVERSITE Louis Pasteur Strasbourg, (1994).

[8] - A. REZEG, S. ACHOUR, II Indice de la minéralisation des eaux dans l'élimination d'acides organiques aromatiques par coagulation Floculation II, Watmed 2Marrakech 14-17 Novembre, (2005)

[9] - L. YOUCEF, S. ACHOUR. "Traitement des eaux fluorées du sud Algérien par combinaison chaux/sulfate d'aluminium II, Watmed 2- Marrakech, 14-17 Novembre, (2005)

[10] - M. MOUNTADAR, S. JAMJAMI, A. NEJEMEDDINE, ॥ Traitement d'un effluent industriel fluoré 11 , déchet - Revue Francophone d'écologie industrielle $\mathrm{N}^{\circ} 30,2^{\text {ème }}$ trimestre, (2003) 25-28

[11] - S. BENZIZOUNE, A. YATRIBI, B. ACHOUR et A. SRHIRI, II Essai d'épuration des eaux usées brutes par une plante aquatique enracinée sous climat tempéré : cas du roseau II, Watmed 2-Marrakech, 14-17 Novembre, (2005)

[12] - S. KHOLTEI, A. BOUZIDI, M. FEKHAOUI, K, R. ANANE, E. E. CREPPY, II Contaminations des eaux souterraines de la plaine Berrechid dans la région de chaouia, au Maroc, par des métaux présents dans les eaux usées II, Effet de la pluviométrie, Vecteur environnement, Volume 36 numér 05, septembre (2000) 68-79.

[13] - R. DESJARDINS, Le traitement des eaux $2^{\text {éme édition }}$

[14] - FICHE technique de Floculant PRAAESTOLR2515.TR, degussa, Stockausen, Division traitement des eaux. 